Deolinda Fontes Cardoso

\title{
Estimação de Canal e Detecção de Sinais em Sistemas CDMA com Múltiplas Portadoras
}

Tese apresentada ao Programa de Pós-graduação em Engenharia Elétrica do Departamento de Engenharia Elétrica da PUC-Rio como requisito parcial para obtenção do título de Doutor em Engenharia Elétrica

Orientador: Prof. Raimundo Sampaio Neto 


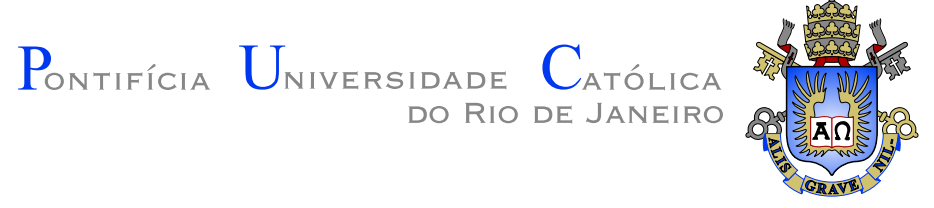

Deolinda Fontes Cardoso

Estimação de Canal e Detecção de Sinais em
Sistemas CDMA com Múltiplas Portadoras

Tese apresentada como requisito parcial para obtenção do título de Doutor pelo Programa de Pós-graduação em Engenharia Elétrica do Departamento de Engenharia Elétrica do Centro Técnico Científico da PUC-Rio. Aprovada pela Comissão Examinadora abaixo assinada.

Prof. Raimundo Sampaio Neto
Orientador
Centro de Estudos em Telecomunicações - PUC-Rio

Prof. Marco Antonio Grivet Mattoso Maia

Centro de Estudos em Telecomunicações - PUC-Rio

Prof. Marcelo Roberto Baptista Pereira Luis Jimenez Centro de Estudos em Telecomunicações - PUC-Rio

Prof. Ernesto Leite Pinto

IME

Prof. Paulo Roberto Rosa Lopes Nunes IME

Prof. Pedro Henrique Gouvêa Coelho

UERJ

Prof. José Eugênio Leal Coordenador Setorial do Centro Técnico Científico

PUC-Rio

Rio de Janeiro, 15 de Dezembro de 2008 
Todos os direitos reservados. É proibida a reprodução total ou parcial do trabalho sem autorização da universidade, do autor e do orientador.

\section{Deolinda Fontes Cardoso}

Graduou-se em Engenharia Elétrica - Eletrônica na Pontifícia Universidade Católica do Rio de Janeiro, adquiriu o título de Mestre em Ciência da Computação pela Universidade Federal Fluminense. Atualmente é Tecnologista em Ciência e Tecnologia no Centro de Analises de Sistemas Navais - CASNAV da Marinha do Brasil.

Ficha Catalográfica

Cardoso, Deolinda Fontes

Estimação de Canal e Detecção de Sinais em Sistemas CDMA com Múltiplas Portadoras / Deolinda Fontes Cardoso; orientador: Raimundo Sampaio Neto. - Rio de Janeiro : PUC-Rio, Departamento de Engenharia Elétrica, 2008.

v., 145 f: il. ; $29,7 \mathrm{~cm}$

1. Tese (doutorado) - Pontifícia Universidade Católica do Rio de Janeiro, Departamento de Engenharia Elétrica.

Inclui referências bibliográficas.

1. Engenharia Elétrica - Tese. 2. Sistema MC CDMA. 3. Sistema MC DS CDMA. 4. Estimação de Canal Assistida e Cega. 5. Decomposição por Valor Singular. 6. Casamento de Correlação. 7. Análise Estatística de Sinais. I. Sampaio-Neto, Raimundo. II. Pontifícia Universidade Católica do Rio de Janeiro. Departamento de Engenharia Elétrica. III. Título. 
Dedico esta Tese a todas as pessoas maravilhosas que me ajudaram a conquistar esta Bem-Aventurança, em especial: ao melhor Professor que eu tive em toda a minha vida; a minha querida família; e aos meus amigos da PUC e do CASNAV-MB. 


\section{Agradecimentos}

Ao Exmo. Sr. Almirante Bernardo José Pierantoni Gambôa, Diretor do Centro de Análises de Sistemas Navais CASNAV-MB, pelo valioso incentivo, apoio e financiamento para o desenvolvimento desta pesquisa. Foi, realmente, muito importante a sua presença para que eu realizasse com sucesso esta etapa da árdua tripla jornada da minha vida: família-trabalho-estudo. Muito Obrigada por tudo o que o Sr. fez para me ajudar! Eu sempre me lembrarei !

Aos meus queridos Pais, José e Natália, pelo seu amor e por terem me ensinado a ser diligente e trabalhadora para conquistar os meus sonhos.

A minha querida família, meu marido e nossos lindos filhos, pelo amor e compreensão sempre presentes, principalmente, durante as minhas longas ausências nestes quatro anos do meu doutorado. Mamãe ama vocês!

Aos meus amigos da PUC, em especial ao Fabian David Backx, um grande amigo muito inteligente e que muito me ajudou nos momentos difíceis. Valeu Fabian, boa sorte para voce!

Aos Professores do Centro de Estudos em Telecomunicações da PUC CETUC, do Departamento de Engenharia Elétrica, Professor Ricardo Prada e Maria Alcina, e aos membros da banca, pela gentileza, cordialidade e contribuições para a finalização desta pesquisa.

Por fim, o maior agradecimento ao meu querido Orientador Professor Raimundo Sampaio Neto, que eu tive a felicidade de ter ao meu lado na vida acadêmica. Muitos anos se passaram desde a minha graduação em Engenharia aqui na PUC, mas não conseguiram apagar da minha lembrança a inesquecível presença do Professor Raimundo com suas aulas primorosas e cuidadosamente preparadas para auxiliar os alunos; listas com exercícios inteligentes por ele próprio elaborados; compreensivo e bondoso; sempre disposto a me ajudar, a me estender a mão nas minhas dificuldades, corrigindo os meus exercícios, tirando as minhas inúmeras dúvidas no desenvolvimento dos modelos matemáticos; acreditando em mim e me fazendo acreditar que eu seria capaz. Muito Obrigada por tudo o que voce representa em minha vida: um Professor brilhante e maravilhoso que guiou, com sabedoria, os meus passos na longa jornada do meu aprendizado. Muito Obrigada, por tudo. Sempre! 


\section{Resumo}

Cardoso, Deolinda Fontes; Sampaio-Neto, Raimundo (Orientador). Estimação de Canal e Detecção de Sinais em Sistemas CDMA com Múltiplas Portadoras. Rio de Janeiro, 2008. 145p. Tese de Doutorado Departamento de Engenharia Elétrica, Pontifícia Universidade Católica do Rio de Janeiro.

Este trabalho investiga o impacto da utilização de técnicas para melhorar a qualidade da estimação de canal em sistemas MC CDMA (Multi Carrier Code Division Multiple Access) e MC DS CDMA (Multi Carrier Direct Sequence Code Division Multiple Access) em canais seletivos em frequência. Dois tipos de intevalos de guarda são considerados: Prefixo Cíclico CP (Cyclical Prefix) e Sufixo de Zeros ZP (Zero Padding) escolhidos por serem os mais populares na literatura. Inicialmente, a estimação de canal é assistida por símbolos piloto e baseada no critério de mínimo erro médio quadrático MMSE (Minimum Mean Square Error). São descritas expressões analíticas para as estimativas convencionais MMSE e para duas técnicas de melhoria das estimativas: aplicando uma matriz de projeção e aplicando uma restrição inicial. Na estimação cega de canal duas técnicas são estudadas para o enlace reverso do sistema MC DS CDMA, incorporando o intervalo de guarda do tipo ZP. A primeira técnica baseia-se na propriedade de ortogonalidade dos subespaços do sinal e do ruído e no método de potências. Na segunda técnica, a estimação de canal é baseada na técnica de casamento de correlação dos dados recebidos e os estimadores são obtidos por minimização de uma função custo. Essa função é definida como o quadrado da norma de Frobenius da matriz de erro resultante da comparação entre a matriz autocorrelação com a sua estimativa obtida por amostragem média. Os resultados simulados demonstraram que os métodos propostos para os algoritmos de estimação assistida e para os algoritmos de estimação cegos apresentam um compromisso desempenho versus complexidade satisfatório, permitem melhorar a qualidade dos respectivos estimadores derivados e revelaram que é possível obter ganho no desempenho de ambos os sistemas.

\section{Palavras-chave}

Sistema MC CDMA. Sistema MC DS CDMA. Estimação de Canal Assistida e Cega. Decomposição por Valor Singular. Casamento de Correlação. Análise Estatística de Sinais. 


\section{Abstract}

Cardoso, Deolinda Fontes; Sampaio-Neto, Raimundo (Advisor). Channel Estimation and Signal Detection in CDMA Systems with Multi Carrier. Rio de Janeiro, 2008. 145p. PhD Thesis - Departamento de Engenharia Elétrica, Pontifícia Universidade Católica do Rio de Janeiro.

This work investigates the impact of techniques to improve the channel estimation in Orthogonal Multi Carrier CDMA (Code Division Multiple Access) Systems: MC CDMA (Multi Carrier Code Direct Multiple Access) and MC DS CDMA (Multi Carrier Direct Sequence Code Division Multiple Access) in frequency-selective channels. Two types of guard interval are considered, the zero padded ( $\mathrm{ZP})$ and cyclic prefix $(\mathrm{CP})$ the most used in the literature. Initially, the channel estimation is assisted by pilot symbols and based in the minimum mean square error criterium. Analytical expressions for the mean square performance of the conventional estimator and two improved channel estimates: applying a projection matrix and applying a initial restriction are presented. In blind channel estimation two techniques are investigate for ZP MC DS-CDMA systems and alternatives methods are proposed. The first is based in subspace method and power techniques. The second is based in correlation matching and square minimization of the cost function; that is defined by the square Frobenius norm of the error matrix. This error matrix is obtained with matching the correlation matrix against its sample average estimated from the received data. The simulated results revel that the proposed assisted and blind methods achieve good performance with softer computational complexity in both systems.

\section{Keywords}

MC CDMA System. MC DS CDMA System. Assisted and Blind Channel Estimation. Singular Value Decomposition. Correlation Matching. Signal Statistics Analysis. 


\section{Sumário}

1 Introdução $\quad \mathbf{1 5}$

1.1 Trabalhos Relacionados 16

$\begin{array}{lll}1.2 & \text { Objetivo } & 18\end{array}$

$\begin{array}{lll}1.3 & \text { Contribuições da Pesquisa } & 20\end{array}$

1.4 Organização do Texto 21

1.5 Notação Adotada $\quad 22$

$\begin{array}{ll}1.6 & \text { Lista de Abreviações } \\ \end{array}$

2 Modelo Vetorial dos Sistemas de Transmissão 24

2.1 DS CDMA 24

$\begin{array}{ll}2.2 \text { OFDM } & 25\end{array}$

2.3 O Canal de Propagação 26

2.4 Os Sistemas Multiportadoras $\quad 29$

$\begin{array}{ll}\text { 2.4.1 MC CDMA } & 29\end{array}$

2.4.2 MC DS CDMA 31

3 Sistema MC CDMA CP: Estimação Assistida, Equalização e Resultados Numéricos

3.1 Modelagem Matemática - Enlace Reverso 34

3.1.1 Média da Estimativa de Canal Convencional 36

3.2 Estimativas de Canal Melhoradas 36

3.2.1 Aplicação da Matriz de Projeção 37

3.2.2 Média da Estimativa de Canal Melhorada 38

3.2.3 Minimização com Restrição Inicial 39

3.3 Análise de Desempenho do Erro Médio Quadrático da Estimativa de Canal $\quad 40$

3.3.1 Perfil Multipercurso do canal 46

3.3.2 Caso particular 1: Códigos Reais Binários 46

3.3.3 Caso Particular 2: Códigos Ortogonais Binários e Sistema com Carga Máxima

3.4 Equalização e Detecção 48

3.5 Resultados Numéricos - MC CDMA CP - Enlace Reverso 49

3.6 Considerações Finais $\quad 55$

3.7 Modelagem Matemática - Enlace Direto 56

3.8 Estimação de Canal Melhorada 57

3.9 Análise de Desempenho do Erro Médio Quadrático da Estimativa de Canal 58

3.10 Estimação com Símbolos e Códigos Pilotos Iguais nos Sinais Piloto

3.11 Equalização e Detecção 60

3.12 Resultados Numéricos - MC CDMA CP - Enlace Direto 60

3.13 Considerações Finais $\quad 63$ 
4 MC CDMA ZP - Estimação de Canal Assistida, Equalização e Detecção e Resultados Numéricos

4.1 Modelagem Matemática - Enlace Reverso 64

4.2 Estimação de Canal Melhorada 65

4.2.1 Aplicação da Matriz de Projeção 65

4.2.2 Minimização com Restrição Inicial $\quad 65$

4.2.3 Média da Estimativa $\quad 66$

4.2.4 Caso Particular 67

4.3 Análise de Desempenho do Erro Médio Quadrático da Estimativa do canal $\quad 68$

$\begin{array}{lll}4.4 & \text { Perfil Multipercurso do Canal } & 71\end{array}$

4.5 Equalização e Detecção 72

4.6 Resultados Numéricos - MC CDMA ZP - Enlace Reverso 74

4.7 Considerações Finais $\quad 76$

4.8 Modelagem Matemática - Enlace Direto 78

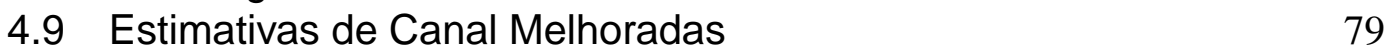

$\begin{array}{lll}\text { 4.9.1 Aplicação da Matriz de Projeção } & 79\end{array}$

4.9.2 Minimização com Restrição Inicial 79

4.9.3 Estimação com Símbolos e Códigos Pilotos Iguais nos Sinais Piloto $\quad 79$

4.10 Equalização e Detecção $\quad 81$

4.11 Resultados Numéricos - MC CDMA ZP - Enlace Direto 82

4.12 Considerações Finais $\quad 84$

5 MC DS CDMA: Estimação Assistida, Equalização e Resultados Numéricos

5.1 Modelagem Matemática - Intervalo de Guarda CP - Enlace Reverso $\quad 85$

5.2 Estimativas de Canal Melhoradas 86

5.2.1 Aplicação da Matriz de Projeção 86

5.3 Modelagem Matemática - Intervalo de Guarda CP - Enlace Direto 87

5.3.1 Estimação com Símbolos e Códigos Pilotos Iguais nos Sinais Piloto

5.4 Equalização e Detecção
5.5 Resultados Numéricos - MC DS CDMA CP - Enlace Reverso e Enlace Direto 90

5.6 Modelagem Matemática - Intervalo de Guarda ZP - Enlace Reverso

$\begin{array}{lll}\text { 5.7 } & \text { Estimativas de Canal Melhoradas } & 96 \\ \text { 5.7.1 } & \text { Aplicação de Matriz de Projeção } & 96\end{array}$

5.7.2 Minimização com Restrição Inicial 96

5.8 Modelagem Matemática - Intervalo de Guarda ZP - Enlace Direto 98

5.8.1 Estimação com Símbolos e Códigos Pilotos Iguais nos Sinais Piloto $\quad 98$

5.8.2 Equalização e Detecção 100

5.8.3 Resultados Numéricos - MC DS CDMA ZP 100

6 Sistema MC DS CDMA ZP: Estimação Cega Baseada em Subespaço e no Método de Potência 
6.1 Métodos de Estimação Cega 106

6.2 Estimação Cega Baseada em Subespaço e no Método de Potência

6.3 A idéia Chave 109

6.4 Equalização e Detecção 112

6.5 Resultados Numéricos - MC DS CDMA ZP - Enlace Reverso 113

6.6 Considerações Finais 118

7 MC DS CDMA ZP - Estimação Cega de Canal Baseada em Casamento de Correlação

7.1 A Técnica de Casamento de Correlação de Dados 119

7.2 Equalização e Detecção 124

7.3 Resultados Numéricos - MC DS CDMA ZP - Enlace Reverso 125

$\begin{array}{ll}7.4 \text { Considerações Finais } & 127\end{array}$

8 Conclusões e Trabalhos Futuros $\quad 131$

8.1 Artigos Publicados em Anais de Congressos Nacionais 133

8.2 Artigos Publicados em Periódicos Nacionais 134

8.3 Artigos Aceitos e Publicados em Simpósios Internacionais 135

$\begin{array}{ll}\text { Referências Bibliográficas } & 136\end{array}$

$\begin{array}{lll}\text { A Apêndice } & 145\end{array}$ 


\section{Lista de figuras}

1.1 Diagrama em Árvore dos Estimadores de Canal Assistidos e Cegos

2.1 Enlace Direto

2.2 Enlace Reverso

2.3 Símbolo OFDM: a) Prefixo Cíclico (CP) b)Sufixo de Zeros Zero Padding (ZP)

2.4 Resposta em Freqüência do Canal $A$

2.5 Resposta em Freqüência do Canal B

2.6 Resposta em Freqüência do Canal B versus Indice da Subportadora

2.7 Resposta em Freqüência do Canal C

2.8 Diagrama em Blocos do Sistema MC CDMA

2.9 Diagrama em Blocos do Sistema MC DS CDMA

3.1 Limitantes Inferior e Superior do Erro Médio Quadrático Normalizado Melhorado e Convencional e Fator de Melhoria do Ruído versus Número de Usuários (K)- MC CDMA CP - Enlace Reverso

3.2 Limitantes Inferior e Superior do Erro Médio Quadrático Normalizado Melhorado e Convencional e Fator de Melhoria do Ruído versus Número de Usuários (K) - MC CDMA CP - Enlace Reverso

3.3 Limitantes Inferior e Superior do Fator de Redução da Parcela da IMA e Fator de Redução da Parcela do Ruido versus Número de Usuários (K) - MC CDMA CP - Enlace Reverso

3.4 Desempenho do Erro Médio Quadrático Normalizado versus (a) $E_{b} / N_{0}$ (b) Número de Usuários (K) - MC CDMA CP Enlace Reverso

3.5 Desempenho da Taxa de Erro de Bit versus $E_{b} / N_{0}-\mathrm{MC}$ CDMA CP - Enlace Reverso

3.6 Fator de Redução da Parcela da IMA versus Carga do Sistema (K/M) - MC CDMA CP - Enlace Reverso

3.7 Desempenho do NMSE versus (a) $E_{b} / N_{o}(\mathrm{~dB})$ (b) Número de Usuários (K) - MC CDMA CP - Enlace Reverso

3.8 Desempenho da Taxa de Erro de Bit versus $E_{b} / N_{o}(\mathrm{~dB})$ Equalização MMSE - MC CDMA CP - Enlace Reverso

3.9 Desempenho Erro Médio Quadrático Normalizado versus (a) $E_{B} / N_{0}(\mathrm{~dB})$ (b) Número de Usuários (K) - Pilotos Ortogonais e Pilotos Iguais - MC CDMA CP - Canal B Randômico Enlace Direto

3.10 Desempenho BER versus Eb/N0(dB) - Simbolos Pilotos Ortogonais e Não-Ortogonais - MC CDMA CP - Canal B Randômico - Enlace Direto 
3.11 Desempenho BER versus Eb/NO(dB) - Simbolos Piloto Ortogonais e Piloto Iguais - MC CDMA CP - Canal B Randômico Enlace Direto

4.1 Limitantes Superiores e Inferiores dos Erros Médios Quadráticos Normalizados (dB) versus (a) $E_{b} / N_{0}$ (dB) (b) Número de Usuários (K) - MC CDMA ZP - Enlace Reverso

4.2 Fatores de Redução da Parcela da IMA e da Parcela do Ruído Aditivo versus Número de Usuários (K) - MC CDMA ZP - Enlace Reverso

4.3 Desempenho do Erro Médio Quadrático Normalizado (dB) versus (a) $E_{b} / N_{0}$ (dB) (b) Número de Usuários (K) - MC CDMA ZP - Enlace Reverso

4.4 Desempenho da Taxa de Erro de Bit versus $E_{b} / N_{0}(\mathrm{~dB})$ - MC CDMA ZP - Enlace reverso

4.5 Desempenho do Erro Médio Quadrático Normalizado (dB) versus (a) $E_{b} / N_{0}$ (dB) (b) Número de Usuários (K) - MC CDMA ZP - Enlace Direto

4.6 Desempenho do Erro Médio Quadrático Normalizado (dB) versus (a) $E_{b} / N_{0}$ (dB) (b) Número de Usuários (K) - MC CDMA ZP - Enlace Direto

5.1 Desempenho do Erro Médio Quadrático Normalizado versus (a) $E_{b} / N_{0}(\mathrm{~dB})$ (b) Número de Usuários (K) - MC DS CDMA CP - Canal C Randômico - Enlace Reverso

5.2 Desempenho da Taxa de Erro de Bit versus $E_{b} / N_{0}(\mathrm{~dB})$ - MC CDMA CP e MC DS CDMA CP - Canal C Randômico - Enlace Reverso $K=16$

5.3 Desempenho da Taxa de Erro de Bit versus $E_{b} / N_{0}(\mathrm{~dB})$ - MC CDMA CP e MC DS CDMA CP - Canal C Randômico - Enlace Reverso $K=32$

5.4 Desempenho de Erro Médio Quadrático Normalizado versus (a) $E_{b} / N_{0}$ (dB) (b) Número de Usuários (K): MC DS CDMA CP Pilotos Iguais e Ortogonais - Canal B Randômico - Enlace Direto

5.5 Desempenho da Taxa de Erro de Bit versus $E_{b} / N_{0}(\mathrm{~dB})$ : MC DS CDMA CP e MC CDMA CP - Pilotos Iguais - Canal B Randômico - Enlace Direto

5.6 Desempenho do Erro Médio Quadrático Normalizado versus (a) $E_{b} / N_{0}$ (b) Número de Usuários (K) - MC DS CDMA ZP Canal B Randômico - Enlace Reverso

5.7 Desempenho do Erro Médio Quadrático Normalizado versus (a) $E_{b} / N_{0}(\mathrm{~dB})$ (b) Número de Usuários (K) - MC DS CDMA ZP - Canal B Randômico - Enlace Direto

5.8 Desempenho do Erro Médio Quadrático Normalizado versus (a) $E_{b} / N_{0}(\mathrm{~dB})$ (b) Número de Usuários (K) - MC DS CDMA ZP - Canal B Randômico - Enlace Direto 
5.9 Desempenho da Taxa de Erro de Bit (BER) versus $E_{b} / N_{0}$ MC CDMA CP e MC DS CDMA CP - Canal B Randômico Enlace Reverso

5.10 Desempenho da Taxa de Erro de Bit (BER) versus $E_{b} / N_{0}$ MC DS CDMA CP e MC DS CDMA ZP - Canal B Randômico - Enlace Reverso

5.11 Desempenho da Taxa de Erro de Bit (BER) versus $E_{b} / N_{0}(\mathrm{~dB})$ - MC DS CDMA ZP e MC CDMA ZP - Canal B Randômico Enlace Reverso

5.12 Desempenho da Taxa de Erro de Bit (BER) versus $E_{b} / N_{0}(\mathrm{~dB})$ - MC DS CDMA ZP e MC CDMA ZP - Canal B Randômico Enlace Direto e pilotos Iguais

6.1 Desempenho de Erro Médio Quadrático versus Número de Blocos de Símbolos Transmitidos $N_{B}$ - MC DS CDMA ZP Canal B Randômico - Enlace Reverso - $E_{b} / N_{0}=10 \mathrm{~dB}$

6.2 Desempenho de Erro Médio Quadrático versus Número de Blocos de Símbolos Transmitidos $N_{B}$ - MC DS CDMA ZP Canal B Randômico - Enlace Reverso - $E_{b} / N_{0}=15 \mathrm{~dB}$

6.3 Desempenho de Erro Médio Quadrático versus Número de Blocos de Simbolos Transmitidos $N_{B}$ - MC DS CDMA ZP Canal B Randômico - Enlace Reverso - $E_{b} / N_{0}=20 \mathrm{~dB}$

6.4 Desempenho de Erro Médio Quadrático versus Número de Blocos de Símbolos Transmitidos $N_{B}$ - MC DS CDMA ZP Canal B Randômico - Enlace Reverso - $E_{b} / N_{0}=25 \mathrm{~dB}$

6.5 Desempenho de Taxa de Erro de Bit (BER) versus Razão Sinal Ruído $E_{b} / N_{0}(\mathrm{~dB})$ - Equalização ZF - MC DS CDMA ZP - Canal B Randômico - Enlace Reverso

6.6 Desempenho de Taxa de Erro de Bit (BER) versus Razão Sinal Ruído $E_{b} / N_{0}(\mathrm{~dB})$ - Equalização MMSE - MC DS CDMA ZP - Canal B Randômico - Enlace Reverso

7.1 Desempenho MSE versus Número de Blocos de Símbolos $N_{B}$ - MC DS CDMA ZP - Enlace Reverso - $E_{b} / N_{0}=5 d B$

7.2 Desempenho MSE versus Número de Blocos de Símbolos $N_{B}$ - MC DS CDMA ZP - Enlace Reverso - $E_{b} / N_{0}=10 d B$

7.3 Desempenho MSE versus Número de Blocos de Símbolos $N_{B}$ - MC DS CDMA ZP - Enlace Reverso - $E_{b} / N_{0}=15 d B$

7.4 Desempenho MSE versus Número de Blocos de Símbolos $N_{B}$ - MC DS CDMA ZP - Enlace Reverso - $E_{b} / N_{0}=20 d B$

7.5 Desempenho BER da Taxa de Erro de Bits (BER) versus $E_{b} / N_{0}(\mathrm{~dB})$ - MC DS CDMA ZP - Enlace Reverso

7.6 Desempenho da Taxa de Erro de Bit (BER) versus Número de Blocos de Símbolos $N_{B}$ - MC DS CDMA ZP - Enlace Reverso 129

7.7 Desempenho da Taxa de Erro de Bit (BER) versus Número de Blocos de Símbolos $N_{B}$ - MC DS CDMA ZP - Enlace Reverso 


\section{Lista de tabelas}

3.1 MC CDMA CP Enlace Reverso - Erro Médio Quadrático Normalizado dos Estimadores Assistidos de Canal

3.2 MC CDMA CP Enlace Direto - Erro Médio Quadrático Normalizado dos Estimadores Assistidos de Canal

4.1 MC CDMA ZP Enlace Reverso - Erro Médio Quadrático Normalizado dos Estimadores Assistidos de Canal

4.2 MC CDMA ZP Enlace Direto - Erro Médio Quadrático Normalizado dos Estimadores Assistidos de Canal

5.1 MC DS CDMA CP Enlace Reverso - Erro Médio Quadrático Normalizado dos Estimadores Assistidos de Canal

5.2 MC DS CDMA CP Enlace Direto - Erro Médio Quadrático Normalizado dos Estimadores Assistidos de Canal

5.3 MC DS CDMA ZP Enlace Reverso - Erro Médio Quadrático Normalizado dos Estimadores Assistidos de Canal

5.4 MC DS CDMA ZP Enlace Direto - Erro Médio Quadrático Normalizado dos Estimadores Assistidos de Canal 